\title{
Comparison of Serum Zinc and Copper Levels in Children with Intractable and Controlled Eplipsy
}

\author{
N.M.El-Nady ${ }^{1}$, A.E.El-Sadek ${ }^{1}$, E.G.Behiry ${ }^{2}$ and S.R.Seif ${ }^{1}$ \\ ${ }^{1}$ Pediatrics Dept., Faculty of Medicine, Benha Univ., Benha, Egypt \\ ${ }^{2}$ Clinical and Chemical Pathology Dept., Faculty of Medicine, Benha Univ., Benha, Egypt \\ E-Mail: Soha11@gmail.com
}

\begin{abstract}
Epilepsy is one of the most common neurological disorders. Epilepsy as the second most burdensome neurologic disorder worldwide in terms of disability-adjusted life years. Copper $(\mathrm{Cu})$ is involved in a number of enzymes with catalase and oxidase-type reactions. This study aimed to identify the role of serum copper in the etiology of epilepsy and its response to treatment Methods: This prospective case controlled study that was conducted on patients attending Outpatient Neurology Clinic and those admitted to Pediatric Department at Benha University Hospital and Benha Children Hospital. Children who participate in this study were classified into two groups; Group I: 60 children with epilepsy. Group II: 60 controls. Serum copper was measured. Results: Copper showed significantly higher level in epileptic cases when compared to control groups $(\mathrm{P}=0.002)$. Copper showed significantly higher level in intractable epileptic cases when compared to controlled subgroups $(\mathrm{p}=0.035)$.Conclusion: Higher cupper levels were significantly associated with epilepsy. higher copper levels were suggested to be independent risk predictors of epilepsy susceptibility.
\end{abstract}

Key words: Copper, Epilepsy, Evaluation.

\section{Introduction}

Seizure disorder is one of the most common neurological diseases in children and occurs at least one time in $4-10 \%$ of children in the first 16 years of life [1].

Occurrence of seizure in children is not only a terrible experience for the parents but also have many neurologic, cognitive, psychological, and social complications for the child and adolescent. Recurrent unprovoked seizures called epilepsy and its diagnosis are done when two or more unprovoked seizures have occurred at intervals longer than 24 hours intervals. The annual prevalence of epilepsy is $0.5-1 \%$ and its lifetime cumulative incidence is $3 \%$. Epilepsy begins in childhood in more than half of the cases. Approximately 50 million people in the world have epilepsy, up to rumrone -third of whom continue to have seizures despite appropriate drug treatment [2].

Despite the importance of identifying refractory epilepsy, due to its comorbidities and sometimes mortalities different definitions presented in the literature. Drug resistant epilepsy may be defined as failure of adequate trials of two tolerated and appropriately chosen and used antiepileptic drugs (AEDs) schedules (whether as monotherapies or in combination) to achieve sustained seizure freedom [3].

The patients categorized as intractable epilepsy when there is failure of seizure control of more than to first line antiepileptic medication with an average of more than one seizure per month for 18 months and no more than three consecutive months seizure free during interval [4].

Copper $(\mathrm{Cu})$ is involved in a number of enzymes with catalase and oxidase-type reactions [5].

This study aimed to identify the role of serum copper in the etiology of epilepsy and its response to treatment.

\section{Patients and methods}

This prospective case controlled study that was conducted on patients attending Outpatient Neurology Clinic and those admitted to Pediatric Department at Benha University Hospital and Benha Children Hospital. All laboratory work was done in Clinical and Chemical Pathology, Benha University Hospital.

\subsection{Subjects}

Children who participate in this study were classified into two groups;

Group I: 60 children with epilepsy

This group was subdivided into two groups:

- Ia- group of intractable epilepsy (Failure of seizure control of more than two first-line antiepileptic medications with an average of more than 1 seizure per month for 18 months and no more than three consecutive months seizure free during that interval)

- Ib-group of controlled epilepsy after two months of treatment by antiepileptic drugs.

Group II: 60 apparently healthy children of the same age, sex and risk factors.

\section{Inclusion criteria for the patients}

1. Epileptic patients.

2. Age 2-15 years of both sexes.

3. At least one-year consumption of AEDs.

\section{Exclusion criteria}

Symptomatic epilepsy due to Central Nervous System infections, metabolic, neurodegenerative disorders and organic lesions. 


\section{Ethical consideration}

An informed consent was obtained from the parents before Enrollment in the study. An approval from Research Ethics Committee in Banha faculty of medicine were obtained.

\subsection{Methods}

All children were subjected to:

- Full medical history

- Full clinical examination

- Laboratory investigations

Serum copper (Blood samples were taken and immediately stored in cool box. Samples were centrifuged, and serum was stored at -20 then were measured by ELISA.)

\section{Statistical analysis}

The collected data was revised, coded, tabulated and introduced to a PC using Statistical package for Social Science (IBM Corp. Released 2011. IBM SPSS Statistics for Windows, Version 20.0. Armonk, NY: IBM Corp.). Data were presented and suitable analysis was done according to the type of data obtained for each parameter.

\section{Descriptive statistics}

1. Mean, Standard deviation $( \pm$ SD) for parametric numerical data, while Median and range for nonparametric numerical data.

2. Frequency and percentage of non-numerical data.

\section{Analytical statistics}

- Student T Test was used to assess the statistical significance of the difference between two study group means.

- Chi-Square test was used to examine the relationship between two qualitative variables

- Fisher's exact test: was used to examine the relationship between two qualitative variables when the expected count is less than 5 in more than $20 \%$ of cells.

- The ROC Curve (receiver operating characteristic) provides a useful way to evaluate the sensitivity and specificity for quantitative diagnostic measures that categorize cases into one of two groups. The optimum cut off point was defined as that which maximized the AUC value.The area under the ROC curve (AUC) results were considered excellent for AUC values between 0.9-1, good for AUC values between 0.8-0.9, fair for AUC values between 0.7-0.8, poor for AUC values between 0.6-0.7 and failed for AUC values between 0.5-0.6.

$\mathrm{P}$ value $>0.05$ is non significant $(\mathrm{N}-\mathrm{S}) \quad \mathrm{P}<0.05$ is significant (S)

\section{Results}

The epilepsy group mean age was 7.2 years $(\mathrm{SD}=2.3)$ years. They were 39 males $(65 \%)$ and 21 females $(35 \%)$. Control group was selected to be matched in age and gender ( $>0.05$ for each). Their mean age was 7.8 years. They were 36 males $(60 \%)$ and 24 females (40\%). Epilepsy group included 30 controlled and 30 intractable cases. No significant differences were found between both subgroups regarding age and gender ( $>0.05$ for age and gender) Table (1).

Cupper showed significantly higher level in epileptic cases when compared to control groups $(\mathrm{p}=0.002)$ Table (2).

Cupper showed significantly higher level in intractable epileptic cases Table (3).

No significant association was found between cupper levels and studied parameters in epilepsy group Table (4).

Receiver operating characteristic (ROC) curve of cupper level was conducted for diagnosis of epilepsy. Cupper showed poor AUC (AUC=0.663), at optimum cut off value $(=105.5)$, sensitivity was $61.7 \%$, specificity was $70 \%$, PPV was $67.3 \%$, NPV was $64.6 \%$ and accuracy was $65.9 \%$ Table (5).

ROC curve of cupper level was conducted for prediction $\mathrm{f}$ intractable epilepsy. Cupper showed poor AUC (AUC=0.639), at optimum cut off value $(=121.5)$, sensitivity was $53.3 \%$, specificity was $93.3 \%$, PPV was $88.8 \%$, NPV was $66.6 \%$ and accuracy was $73.3 \%$ Table (6).

Table (1) Comparison of demographic data between studied groups and subgroups.

\begin{tabular}{|c|c|c|c|c|c|c|c|c|c|c|c|}
\hline & \multirow[b]{3}{*}{ Mean \pm SD } & \multirow{2}{*}{\multicolumn{2}{|c|}{$\begin{array}{c}\text { Control } \\
\mathbf{N}=60\end{array}$}} & \multirow{2}{*}{\multicolumn{2}{|c|}{$\begin{array}{c}\text { Epilepsy } \\
\mathrm{N}=60\end{array}$}} & \multirow[t]{2}{*}{$\mathbf{P}$} & \multirow{2}{*}{\multicolumn{2}{|c|}{$\begin{array}{c}\text { Controlled } \\
\mathrm{N}=\mathbf{3 0}\end{array}$}} & \multirow{2}{*}{\multicolumn{2}{|c|}{$\begin{array}{c}\text { Intractable } \\
\mathrm{N}=\mathbf{3 0}\end{array}$}} & \multirow[t]{2}{*}{$\mathbf{P}$} \\
\hline & & & & & & & & & & & \\
\hline Age & & 7.8 & 2.6 & 7.2 & 2.3 & 0.263 & 7.9 & 2.6 & 6.5 & 2.1 & 0.182 \\
\hline Males & $\mathrm{N}, \%$ & 36 & $60 \%$ & 39 & $65 \%$ & 0.572 & 20 & $66.7 \%$ & 19 & $63.3 \%$ & 0.787 \\
\hline Females & $\mathrm{N}, \%$ & 24 & $40 \%$ & 21 & $35 \%$ & & 10 & $33.3 \%$ & 11 & $36.7 \%$ & \\
\hline
\end{tabular}

Numerical data are expressed as mean and SD, compared by independent $t$ test; categorical data are expressed as number and percentage; compared by chi square test. 
Table (2) Comparison of cupper level between cases and control groups.

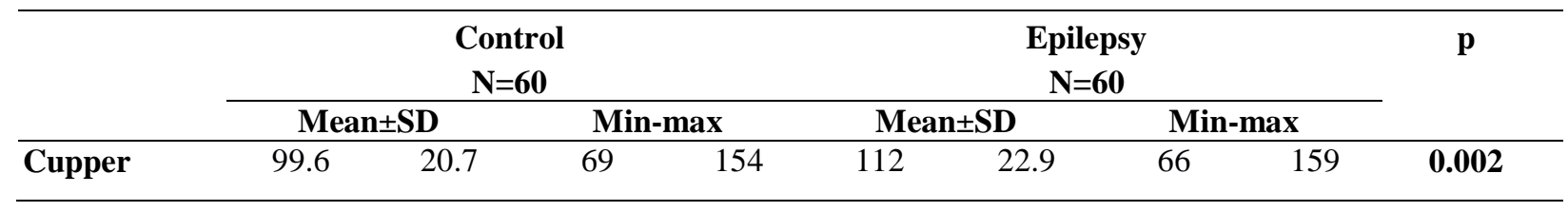

Numerical data are expressed as mean and SD, compared by independent $\mathrm{t}$ test.

Table (3) Comparison of cupper levels between cases subgroups.

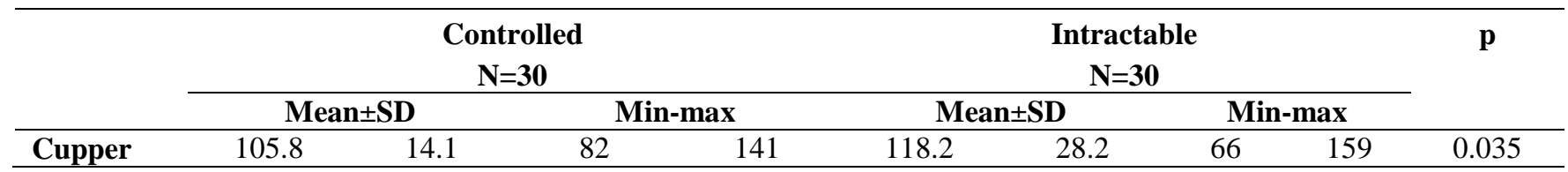

Numerical data are expressed as mean and SD, compared by independent $\mathrm{t}$ test.

Table (4) Association of cupper levels according to studied parameters in epilepsy group.

\begin{tabular}{|c|c|c|c|c|c|}
\hline & & \multicolumn{4}{|c|}{ cupper } \\
\hline & & Mean & \pm & SD & p \\
\hline \multirow[t]{2}{*}{ Gender } & Male & 109.6 & \pm & 24.2 & 0.281 \\
\hline & Female & 116.4 & \pm & 20.1 & \\
\hline \multirow[t]{2}{*}{ Family history of epilepsy } & Negative & 116.5 & \pm & 22.3 & 0.107 \\
\hline & Positive & 106.4 & \pm & 19.8 & \\
\hline \multirow{2}{*}{ Consanguinity } & Negative & 110.6 & \pm & 23.0 & 0.365 \\
\hline & Positive & 117.4 & \pm & 23.0 & \\
\hline \multirow[t]{2}{*}{ Perinatal insult } & Negative & 112.7 & \pm & 20.6 & 0.702 \\
\hline & Positive & 110.2 & \pm & 28.7 & \\
\hline \multirow[t]{2}{*}{ Neonatal Seizure } & Negative & 113.5 & \pm & 22.1 & 0.391 \\
\hline & Positive & 107.8 & \pm & 25.3 & \\
\hline \multirow[t]{2}{*}{ Developmental milestones } & Normal & 112.1 & \pm & 20.5 & 0.982 \\
\hline & delayed & 111.9 & \pm & 24.6 & \\
\hline \multirow{2}{*}{ Power } & Normal & 111.3 & \pm & 22.3 & 0.725 \\
\hline & Decreased & 113.6 & \pm & 25.0 & \\
\hline \multirow[t]{3}{*}{ Tone } & Normal tone & 111.7 & \pm & 19.9 & 0.991 \\
\hline & Hypotonia & 112.7 & \pm & 25.4 & \\
\hline & Hpertonia & 112.6 & \pm & 28.3 & \\
\hline \multirow[t]{3}{*}{ Reflexes } & Normal & 109.6 & \pm & 20.5 & 0.106 \\
\hline & Hyporeflexia & 129.5 & \pm & 14.0 & \\
\hline & Hyperreflexia & 116.9 & \pm & 26.0 & \\
\hline \multirow{2}{*}{ Cranial nerve palsy } & Absent & 112.3 & \pm & 22.1 & 0.890 \\
\hline & Present & 111.3 & \pm & 25.8 & \\
\hline \multirow[t]{2}{*}{ Speech } & Normal & 108.9 & \pm & 21.2 & 0.257 \\
\hline & Delayed & 115.7 & \pm & 24.7 & \\
\hline \multirow[t]{2}{*}{ Mentality } & Normal & 111.2 & \pm & 21.0 & 0.780 \\
\hline & Low & 112.9 & \pm & 25.5 & \\
\hline \multirow[t]{5}{*}{ EEG } & Normal & 110.0 & \pm & 22.2 & 0.905 \\
\hline & Focal epileptogenic activity & 116.4 & \pm & 22.9 & \\
\hline & Generalized epileptogenic activity & 112.0 & \pm & 23.4 & \\
\hline & Multifocal epileptogenic activity & 115.0 & \pm & 29.9 & \\
\hline & Hysarrthymia & 97.0 & & & \\
\hline
\end{tabular}

$\mathrm{SD}$, standard deviation; independent $\mathrm{t}$ test was used for comparison between 2 groups; ANOVA was used for comparison between more than two groups. 
Table (5) Validity of copper level for diagnosis of epilepsy.

\begin{tabular}{lc}
\hline & Cupper \\
\hline AUC (95\% CI) & $0.663(0.565-0.761)$ \\
Cut off & 105.5 \\
Sensitivity (\%) & 61.7 \\
Specificity (\%) & 70 \\
PPV (\%) & 67.3 \\
NPV (\%) & 64.6 \\
Accuracy (\%) & 65.9 \\
\hline
\end{tabular}

AUC, area under ROC, receiver operating characteristic curve; CI, confidence interval; PPV, positive predictive value; NPV, negative predictive value.

Table (6) Validity of copper level for prediction of failure of treatment (intractable epilepsy).

\begin{tabular}{lc}
\hline & Cupper \\
\hline AUC & 0.639 \\
95\% CI & $0.488-0.791$ \\
Cut off & 121.5 \\
Sensitivity (\%) & 53.3 \\
Specificity (\%) & 93.3 \\
PPV (\%) & 88.8 \\
NPV (\%) & 66.6 \\
Accuracy (\%) & 73.3 \\
\hline
\end{tabular}

AUC, area under ROC, receiver operating characteristic curve; CI, confidence interval; PPV, positive predictive value; NPV, negative predictive value.

\section{Discussion}

This study showed that, copper showed significantly higher level in epileptic cases when compared to control groups $(\mathrm{P}=0.002)$. Copper showed significantly higher level in intractable epileptic cases when compared to controlled subgroups $(\mathrm{p}=0.035)$.

This agreed with Wojciak et al., [6] who found serum copper level of epileptic patients were higher than healthy group and they recommended that epilepsy may increase the copper level.

This was observed by other authors Prasad et al., [7] that have observed higher levels of serum $\mathrm{Cu}$ in epilepsy patients than in the control group.

Serum $\mathrm{Cu}$ levels in epileptic patients were significantly higher than the control group that is consistent with other studies [8,7] and this was attributed to the effect of AEDs, increased hepatic synthesis, or due to the decreased breakdown or both $\mathrm{Cu}$ binding proteins, altered intestinal absorption, and altered excretion patterns, changes in the distribution among body tissues, or a combination of these factors. Also, these results are in agreement with a study carried out by Sarangi et al., [9] who found statistically significant increase serum $\mathrm{Cu}$ as enzyme - inducing potential of anti-epileptic drugs leading to increase in hepatic synthesis of ceruloplasmin has been suggested as a possible mechanism.

Studies regarding the results of $\mathrm{Cu}$ concentration in epilepsy are contradictory where,

Nazıroğlu and Yürekli, [10] found that depleted blood copper level may be associated with the pathogenesis of epilepsy.
This was in disagreement with Saad et al., [11] who studied trace elements, oxidant, and antioxidant enzyme values in blood of children with refractory epilepsy in comparison to healthy control children and found no statistical difference in $\mathrm{Cu}$ among patient group than those in the control group.

Elshorbagy et al., [12] who found that serum copper levels were not significantly lower in cases than controls.

Also, this was in disagreement also with Eissa et al., [13] who found no statistical significance on measuring the copper serum level between patients group when compared to the control group.

Our results were in disagreement with Kheradmand et al., [14] who found there was no statistically significant difference between serum copper levels of intractable and controlled epilepsy group ( $\mathrm{P}$ value $=0.811$ ), although $48.58 \%$ of the intractable epilepsy group and 45.72 of the controlled epilepsy group were copper deficient.

This study showed that, no significant association was found between cupper levels and studied parameters in epilepsy group.

This was in agreement with Eissa et al., [15] who found no significant difference was found between copper serum levels of newly diagnosed, untreated patients, and previously diagnosed, and treated ones, this was opposite to Saboktakin et al., [8] findings as they found that serum copper was significantly higher in treated patients' group than in the untreated group. Eissa et al., [15] also found that the serum level of copper showed no significant difference between patients on 
monotherapy and others on polytherapy. Again, other studies found that the serum copper was higher in patients on polytherapy treatments compared in patients on monotherapy $[15,16]$. All these studies included patients on phenytoin antiepileptic drug that was accused as the main cause of elevated serum copper in treated patients' group, yet our study did not include any patient with phenytoin intake.

Eissa et al., [15] did not find any significant difference in copper serum level between patients on either carbamazepine or levetiracetam on hand and patients who were not on these medications and control on the other hand.

Eissa et al., [15] in their study found, patients with past history of febrile seizures had no significant difference in their copper serum level in comparison to those without febrile seizures in their past history.

\section{Conclusion}

Higher cupper levels were significantly associated with epilepsy suscebtability, with cupper sensitivity of $53.3 \%$, specificity of $93.3 \%$.

\section{References}

[1] EC.Stafstrom, \& L.Carmant, Seizures and epilepsy: an overview for neuroscientists. Cold Spring Harbor perspectives in medicine, Vol.5, PP.022-426,2015.

[2] W.Farghaly, MA.Abd Elhamed, E.Hassan, Prevalence of childhood and adolescence epilepsy in Upper Egypt (desert areas). Egypt J Neurol Psychiatry Neurosurg, Vol.54, PP.3-4,2018.

[3] F.López González, X.Rodríguez Osorio, A.GilNagel Rein, M.Carreño Martínez, J.Serratosa Fernández, V.Villanueva Haba, J.Mercadé Cerdá, MDrug-resistant epilepsy: Definition and treatment alternatives. Neurología (English Edition), Vol.30, PP.439-446,2015.

[4] F.Tang , A.Hartz, S.M, \& B.Bauer ,Drug-Resistant Epilepsy: Multiple Hypotheses, Few Answers. Frontiers in Neurology, Vol. 99, PP.7-8,2017.

[5] I.Wessels, M.Maywald, \& L.Rink, as a Gatekeeper of Immune Function. Nutrients, Vol.9,12-86,2017.

[6] RW.Wojciak, E.Mojs, M.Stanislawska-Kubiak, The serum zinc, copper, iron, and chromium concentrations in epileptic children. Epilepsy Res, Vol.104, PP.40-44,2013.
[7] R.Prasad, A.Singh, BK.Das,Cerebrospinal fluid and serum Zinc, Copper, Magnesium and Calcium levels in children with idiopathic seizure. Journal of Clinical and Diagnostic Research, Vol.3, PP.1841$1846,2009$.

[8] L.Saboktakin, M.Barzegar, Jo.Hagh, M.Emamalizadeh,Study on serum Copper and Zinc level of children with epilepsy during long term therapy with anticonvulsants. Life Sci J, Vol.9, PP.1250-1254,2012.

[9] SC.Sarangi, M.Tripathi, AK.Kakkar, Effect of antiepileptic therapy on trace elements status in Indian population in a tertiary care hospital from northern India: a cross sectional study. Epilepsy Res , Vol.108, PP.917-927,2014.

[10] M.Nazıroğlu,VA.Yürekli, Effects of antiepileptic drugs on antioxidant and oxidant molecular pathways: focus on trace elements. Cell Mol Neurobiol, Vol.33, PP.589-599,2013.

[11] K.Saad, E.Hammad, AF.Hassan, Trace element, oxidant, and antioxidant enzyme values in blood of children with refractory epilepsy. Int J Neurosc, Vol.124, PP.181-186,2014.

[12] H.Elshorbagy, M.Bassiouny, N.Kamal, Study of Trace Elements and Role of Zinc Supplementation in Children with Idiopathic Intractable Epilepsy. Journal of Pediatric Epilepsy, Vol.05, PP.026$033,2016$.

[13] M.A.Eissa, K.O.Abdulghani ， M.A.Nada, Serum zinc and copper levels in a sample of Egyptian epileptic children. Egypt J Neurol Psychiatry Neurosurg, Vol.56, PP.7-9,2020.

[14]Z.Kheradmand, B.Yarali, A.Zare,Comparison of serum zinc and copper levels in children and adolescents with intractable and controlled epilepsy. Iran J Child Neurol, Vol.8, PP.49-54,2014.

[15]H.Ulvi, R.Yigiter, T.Yoldas, Y.Dolu, A.Var, B.Mungen, Zinc and copper contents in hair and their serum concentrations in patients with epilepsy. Eastern J Med, Vol.7, PP.31-5,2002.

[16] SI.Helal, HS.Megahed, SM.Salem, ER.Youness, Monotherapy versus polytherapy in epileptic adolescents. Maced J Med Sci, Vol.6, PP.1747,2013 . 